\title{
BREVE SEMBLAZA Y RECUERDO DEL PROFESOR CIFUENTES DELATTE, COMO MIEMBRO DE LA REAL ACADEMIA NACIONAL DE MEDICINA
}

\author{
Juan-Evangelista Ruiz de Burgos Moreno \\ Real Academia Nacional de Medicina. Madrid. España.
}

\begin{abstract}
Resumen.- Durante mi formación en la Clínica Universitaria de Urología de Munich y en el Hospital GroBhodern, pude comprobar el prestigio que tenía el Dr. Cifuentes Delatte entre mis maestros, que además de conocerle personalmente, hacían a menudo referencia, a sus trabajos científicos. Detallo la ocasión en que le conocí durante mi ingreso como Académico Correspondiente de la Real Academia de Medicina y las conversaciones que tuvimos en las sesiones de la Academia, así como la amistad que terminé teniendo con él.
\end{abstract}

Palabras clave: Real Academia Nacional de Medicina. Luis Cifuentes Delatte.

Summary.- During my training in the University Clinic of Urology in Munich and Gro-Bhodern Hospital I could realize the prestige Dr. Cifuentes Delatte had among my Professors, who in addition to knowing him personally made frequent references to his scientific works. I write in detail the time I met him during my admission in the Royal Academy of Medicine as a Correspondent Academician and the conversations we had during the sessions of the Academy, as well as the friendship I finally had with him.
Keywords: National Royal Academy of Medicine. Dr. Luis Cifuentes Delatte.

\section{INTRODUCCIÓN}

Conocía al Profesor Cifuentes, como muchos de mis compañeros urólogos, a través de sus libros. En los años ochenta habían poco libros en español que describieran un abordaje tan descriptivo de la resección transuretral como lo hacía el Prof. Cifuentes en su libro: "Cirugía urológica endoscópica", cuya primera edición fue realizada en 1961, y la última en 1980. Su lectura y estudio era casi obligada y necesaria para todos nosotros por aquel entonces, y es qué, con buen juicio crítico y con el pasar de los años, comentaba D. Luis qué en dicho libro había intentado plasmar con todo el entusiasmo docente todo lo que conocía de la cirugía endoscópica, porque:

"...no existía la oportunidad de la que hoy se pueden aprovechar muchas clínicas, de poder contemplar todo tipo de intervenciones endoscopicas mediante su proyección en una pantalla. Con ello habrá desaparecido el hermetismo que en un principio tuvo esta cirugía y que dificultó su enseñanza y su justa apreciación".

Pero el profesor Cifuentes aparte de ser pionero de la cirugía endoscópica en España, fue un destacado investigador de las "cistitis y cistopatías", realizando las primeras enterocistoplastias por tuberculosis en nuestro país. Su doble faceta de clínico e investigador la ejerció en todo su quehacer urológico y especialmente en el campo de la urolitiásis, realizando diversas aportaciones. Así lo podemos comprobar en las numerosas comunicaciones y presentaciones realizadas a la Real Academia, y recogidas en los Anales de la misma.

Durante los casi seis años de mi formación en Alemania fueron varias las ocasiones en las que pude comprobar el prestigio que el Prof. Cifuentes tenía. Mis profesores le conocían. El profesor Schmiedt, 
jefe de la clínica universitaria de Urología de Munich, me dijo un día en la sala de endoscopia del Hospital GroBhadern que conocía al profesor Cifuentes y sus publicaciones en el campo de la litiásis. También el profesor Gerhard Staehler, jefe clínico de GroBhadern, me hacia referencias a él, pues mantuvo intercambios sobre la utilización endoscópica del láser YAG-Nd de la $M B B$, primer láser urológico para el tratamiento de pequeños tumores vesicales, y sobre el que preparamos un trabajo que luego publicamos también en España e hicimos referencia al Profesor Cifuentes. El profesor Christian Chaussy que desarrollaba la aplicación de las ondas de choque conocía sus trabajos sobre etiopatogénia de la litiásis. En fin, qué durante los casi tres años que permanecí en Munich, la nutrida representación de urólogos de todo el mundo que pasó por allí, para conocer el método ESWL, me dejó claro qué el profesor Cifuentes era un referente de la urología española a nivel internacional.

Pero la ocasión de conocer personalmente al Profesor Cifuentes la tuve durante mi ingreso como Académico Correspondiente en la Real Academia Nacional de Medicina, en 1989, y fue a propósito de mi comunicación a la misma, sobre la ESWL, titulada "Litofragmentación Renal Extracorpórea: desarrollo y aplicación actual". Tras la misma don Luis pidió la palabra para decir: "Mi felicitación más cordial al Dr. Juan Evangelista Ruiz de Burgos Moreno, por su presentación de un método terapéutico verdaderamente revolucionario y de enorme y auténtica eficacia sobre el que hacia hablar aquí en la Academia... El urólogo decía yo no debe ser un simple litotomista frente a la litiasis. Los urólogos son ya poco litotomistas, felizmente, pero tampoco deben quedarse en litofragmentadores, litotriptores. He advertido un menor interés en la investigación de la fisiopatología de la litiasis, y esto a nivel universal, desde que los cálculos se destruyen tan fácilmente. Soy entusiasta decidido de la litotricia renal extracorpórea, y me alegro de veras que se haya reducido el numero de intervenciones quirúrgicas de esta afección, pero no olvidemos la gran importancia que sigue teniendo el básico de lo que es la litiasis renal en un plano general y en el plano concreto y personal de cada enfermo, pues la reiteración de la sesiones de ondas de choque, repito, no creo que sea inocua.

Mi felicitación, otra vez, a Juan Evangelista, que haciendo honor a su nombre, nos ha traído también una buena nueva".

Don Luis Cifuentes Delatte ingresó el 30 de Mayo de 1972 como Académico Numerario en la Real Academia Nacional de Medicina. Su discurso del Acto de Recepción se titulaba "Origen y desarrollo de los cálculos renales". Al principio del mismo dice D. Luis:
"...El día de hoy tiene una doble significación, una personal por lo que este acto significa y que dependiente de mi seguirá siendo fiel a la dignidad del médico, al amor a nuestra profesión y a los fundamentos de nuestro arte y ciencia que guiaron en su noble esfuerzo a los egregios fundadores de nuestra Academia. La otra vertiente se inclina hacia el pasado. Recuerdo aquella tarde de diciembre, hace ya muchos años, en la que yo, joven estudiante de medicina, entré por primera vez en esta Casa y me senté entre el publico como hijo del nuevo Académico que aquel día leía su discurso de ingreso. La vida me ha llevado, proseguía a la misma profesión, a la misma especialidad, y hoy me trae también a esta Academia y no oculto la satisfacción que me produce haber conseguido este gran honor que en aquel tiempo mereció mi padre. Su extraordinaria labor en cirugía urológica le hizo acreedor a tal reconocimiento, pero mis elogios podrían ser considerados parciales, por los que renuncio a ellos, aunque no al emocionado recuerdo y al homenaje intimo a quien en su vida, estuve tan unido con el doble afecto de hijo y discípulo. El tiempo ha pasado, pero veo este Salón, esta tarde, como aquel día, con una elegante solemnidad".

Así evocaba don Luis el recuerdo de su padre, D. Pedro Cifuentes y Díaz, que junto a los Drs. González Bravo, De la Peña y Negrete fundó en 1910

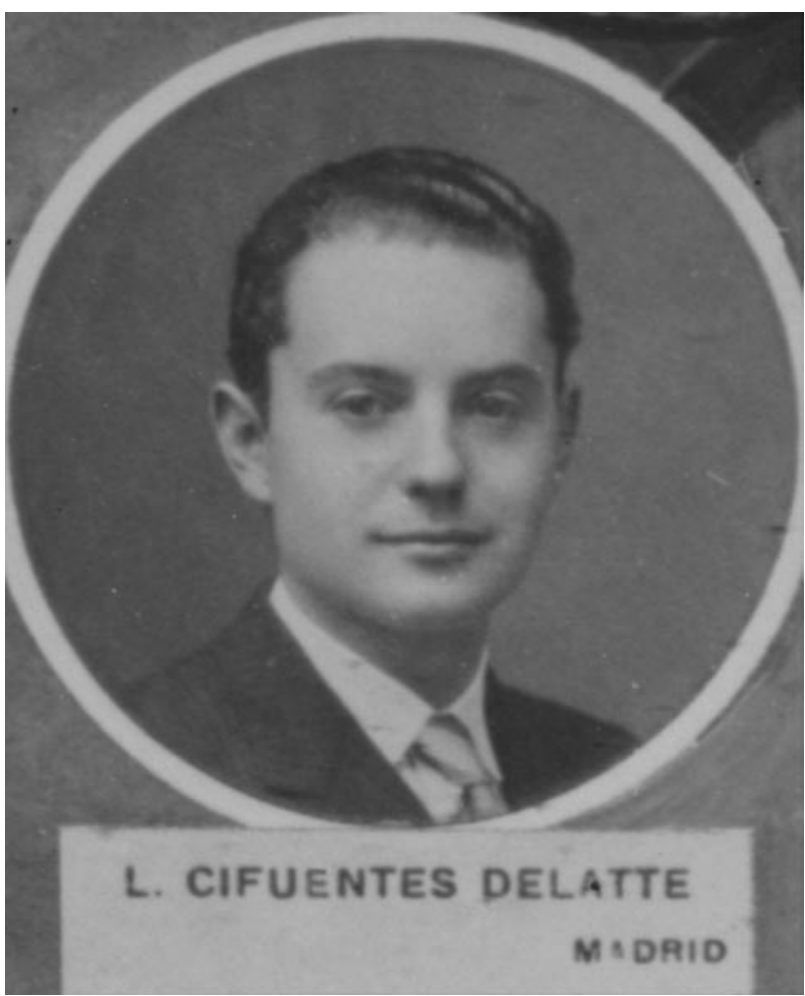

FIGURA 1. Profersor Cifuentes Delatte. 
la Asociación Española de Urología, y que ocupó el sillón numero ocho en 1929, por vacante del Dr. Isla.

Don Luis sentía con emoción la Academia. Realizó comunicaciones a la misma hasta casi el final de su vida, mientras tuvo condiciones para hacerlo. Conocía bien su historia, desde el modesto origen tertuliano que tuvo reuniéndose periódicamente médicos, cirujanos y farmacéuticos en la rebotica de la Oficina de Farmacia de D. José Ortega de la calle de la Montera número 19, y que el 12 de julio de 1773 se le intenta dar carácter oficial con el nombre de Tertulia Literaria Medica Matritense. Este era el pequeño núcleo que el 12 de agosto de 1734 se convertiría por Real Decreto de Felipe $V$ en Real Academia Médica. Pues bien, en 1972, como hemos dicho, D. Luis ingresó en la Real Academia Nacional de Medicina, perteneciente al Instituto de España para ocupar el sillón número catorce, por vacante de D. Alfonso de la Peña Pineda, $y$ en su discurso le recuerda, al igual que lo hace con su padre, y con otros antecesores inmediatos con estas palabras:

"Este Sillón ha sido ocupado por figuras destacadas como eran, entre las correspondientes a este siglo, el doctor Francisco Huerta y el doctor Santiago Castro. Sucedo directamente a un compañero de la especialidad, el doctor Alfonso de la Peña. Recuerdo muy bien la primera vez que le ví. Yo acababa de terminar mis estudios en la Facultad cuando el volvía de los Estados Unidos de completar sus estudios en la Clínica Mayo. Traía ya la experiencia de los procedimientos endoscópios. Estoy viendo, con los ojos del recuerdo, a Alfonso de la Peña, teniendo en sus manos, en una sesión de la Asociación Española de Urología, el resectoscopio de corte frío... Desde aquel día ambos seguimos labores en distintos centros hospitalarios, pero en los contactos esporádicos mantenidos pude comprobar cuan grande es la fidelidad de sus discípulos y el recuerdo emocionado que todos guardan de su Maestro".

Prosiguió haciendo lo propio con otro maestro importante en su vida como bien sabemos, don Carlos Jiménez Díaz, a quien, entre tantas cosas, evoca:

"...le debo la llama que prendió en mí del amor a la investigación clínica y a cuya memoria quiero también rendir un homenaje cordial y afectuoso".

Con Jiménez Díaz fue alumno interno tras finalizar su carrera, y bajo su dirección defendió su tesis doctoral en 1935; "El equilibrio ácido base en las nefropatías". A él dedico su libro "Composición y estructura de los cálculos renales" (1984), con el siguiente epígrafe: "A memoria del Profesor Carlos Jiménez Díaz, que supo despertar en tantos de noso- tros el amor a la investigación clínica". Cifuentes era realmente un emocionado defensor practicante de la noble relación maestro y discípulo, y siempre que se hablaba con él se apreciaba su entusiasmo, su disposición y su calidad docente. Ese reconocimiento a aquellos de los qué él aprendió y a que aquellos que con él y de él aprendieron fue algo que acompaño siempre al profesor Cifuentes Delatte. Supo recibir como discípulo y supo dar y transmitir como maestro. Es pues, parafraseando a Laín Entralgo de su apotegma, el buen maestro, quien en algún momento de su vida sabe ser discípulo de sus discípulos, y buen discípulo quien, en algún momento de su vida, sabe ser maestro de sus maestros.

Pero también su calidad humana la comprobamos por ejemplo, en la comunicación que hace a la Real Academia en 1995 del que fuera también maestro suyo en cirugía general y acogió como ayudante durante la guerra civil, en el improvisado hospital del colegio Nuestra. Sra. del Pilar de Madrid; "Un magnifico cirujano español, D. Plácido González Duarte, 1897- 1986". Es por ello que, el profesor Gracia Guillén, en el discurso de precepto a su memoria dice; "...que no sólo fué un excelente clínico y un magnífico investigador, Luis Cifuentes fue una gran persona", y destacaba de su vida el entusiasmo que hacia referencia como constante de su vida, aprendido de su maestro y gran amigo Xavier Zubiri.

Su calidad docente era ciertamente algo prosaica porque era muy descriptiva, como lo demuestra en sus libros y trabajos, algo sin duda favorecido por una estupenda memoria. Relataba casos clínicos como si hubiera visto ayer al paciente. Veamos por ejemplo como describe la patocrónia de un caso, comunicándolo en la Academia;

"...aquella enferma con un cálculo que le llenaba prácticamente la pelvis renal y que normalmente tendría una clara indicación operatoria en aquella época, no tan remota, puesto que de aquello nos separan tan sólo unos diecisiete años. El estado general de esta paciente no era demasiado bueno y pensé que era absolutamente aconsejable iniciar en ella un tratamiento con zumo de limón, incrementando con un simple medicamento a base de bicarbonato y citratos sódicos. No abrigaba demasiada esperanza en cuanto a la posible disolución del cálculo, pero la enferma iba a ensayar aquel tratamiento y volver a los dos meses para ser observada de nuevo $y$, si su estado general había mejorado, probablemente para ser intervenida. La enferma se fue encontrando mucho mejor tras aquellas tomas de zumo de limón y de alcalinos, sus molestias renales y su hematuria fueron lentamente disminuyendo. Tal mejoría y otras circunstancias personales de la enferma, se opusieron a un 
nuevo viaje desde su ciudad de Castilla La Vieja. Se encontraba tan bien con aquel tratamiento que no lo interrumpió durante un año, hasta que vino a decirme que se encontraba perfectamente. La exploración urográfica demostró la total desaparición de su cálculo. No tenía el más pequeño síntoma renal y la hematuria había desaparecido por completo, siendo negativo el estudio microscópico del sedimento".

Fueron muchas las veces que coincidí con don Luis en las sesiones científicas de la Real Academia, y fácilmente me comentaba anécdotas de su estancia en la Alemania, a la sazón de mi estancia en dicho país. Me contaba como era entonces la vida hospitalaria, allá por 1930, en Berlín, junto al famoso profesor von Lichtenberg, y de su aprendizaje de la cateterización uretral. Me entretenía el escucharle porque su lenguaje descriptivo, como hemos dicho, le hacía ser algo novelado y así, ofreciendo buen lujo de detalles en su exposición, se podía imaginar fácilmente como era todo, una lección de turismo histórico.

Una vez de las qué coincidí con $D$. Luis en la Academia fue el 3 de abril de 1990, estuvimos hablando tras la sesión sobre la valoración actual de las cistitis en la mujer. Le acompañe a su casa y quede otro día para que me firmara dos de sus libros; "Estructura y composición de los cálculos renales" y "Cistitis y Cistopatías". Me escribió una dedicatoria muy amable que le agradecí. Me acuerdo que al final me comentó, no sin cierto aire de nostalgia, sobre la gran evolución que se avecinaba en la Medicina en los próximo años, de las ventajas y desventajas de una Medicina excesivamente tecnificada, pero que iba a ser sorprendente... No en vano había leído el discurso de la Solemne Sesión Inaugural del curso de 1986 de la Real Academia Nacional de Medicina, celebrada el 14 de enero, con el título: "Comentarios a la evolución de la Urología". Hablar él de futuro era hablar de investigación. En dicha comunicación, por ejemplo enfatizaba el desarrollo de nuestra especialidad cuando se conjugaba clínica e investigación'. Esto era algo que promulgó y practicó ejemplarmente durante su vida profesional. Al hablar sobre cirugía y litiásis urinaria en la Academia, en 1972, decía:

"...constituye una ocupación frecuentísima de los urólogos, casi diaria, la tarea quirúrgica de extraer cálculos por diversas técnicas operatorias. Pero si yo insisto en el conocimiento básico de la litiasis, es, precisamente porque ello representa una inquietud que proviene de la preocupación por nuestros propios enfermos, que surge en nuestros quirófanos, que es inseparable e nuestra labor de cirujanos, He dicho en más de una ocasión, que el urólogo actual no puede ser frente a la litiasis tan sólo como fueron aquellos sus antepasados, aquellos viejos litotomistas que recorrían Europa extra- yendo piedras de veiigas humildes o ilustres y admirándose siempre de aquel misteriosos mal, acerca de cuyo origen se especulaba mas con citas galénicas que con la propia observación... Por complejo que ello sea es al urólogo al que compete no solo el manejo del bisturí sino también con ello el conocimiento profundo de la fisiopatología de la litiasis..., evitar la recidivas será fruto del esfuerzo investigador".

La última vez que vi a don Luis fue el 24 de noviembre de 1993, en una sesión de la Academia, al término de la misma quise saludarle para hablar con él de su articulo publicado en Archivos Españoles de Urología, con el título de "Mi aprendizaje de la cirugía endoscópica" describiendo su estancia en Estados Unidos en 1946, porque de su lectura, nostálgica y cargada con la emoción de un recuerdo singular, donde se destilan los valores médico, científicos y humanos que hemos comentado y que el tuvo la suerte de ejercer y compartir durante su vida con ejemplaridad.

El día 5 de Abril del 2005 se celebró Sesión Neurológica en memoria de D. Luis Cifuentes Delatte, con un discurso de precepto por el Académico D. Luis Gracia Guillén. En su alocución dijo: "... era maestro en muchas cosas de la vida, pero en el campo profesional maestro en Urología. Luis Cifuentes ha sido un gran urólogo. Y ello, en las dos vertientes de esta disciplina, la clínica y la científica. En este orden preciso, porque Luis Cifuentes fue un médico de pies a cabeza y se vió a si mismo siempre como servidor de enfermos. La ayuda depurada al enfermo le llevó a investigar. De ahí que el clínico se tornara en investigador. Su camino fue de la clínica a la investigación y de la investigación a la clínica".

Igualmente D. Pedro Laín Entralgo decía también en la Academia qué: "... Luis Cifuentes había conseguido el paso responsable del cirujano, de los años de aprendizaje, y los años de peregrinación a los años de magisterio. Pero no se había permitido, como exige la Medicina actual, dejar de ser aprendiz y peregrino como quien quiere seriamente practicar y hacer una buena Medicina, aunque con toda la legitimidad con la que haya llegado a ser un verdadero maestro".

Si Cicerón escribió que la vida de los muertos esta depositada en la memoria de los vivos, no hay duda que somos muchos los médicos y urólogos que mantenemos a don Luis Cifuentes presente en nuestra memoria. Y si el emblema de la Real Academia Nacional de Medicina, escrito alrededor de esa composición especular parabólica de Arquímedes para concentrar los rayos solares, es: "Major collectis viribus exit", no hay duda de que don Luis Cifuentes fue un Miembro que le dio honor y engrandecimiento a la Institución. 